\title{
Optomechanical cantilever device for displacement sensing and variable attenuator
}

\author{
Peter A. Cooper*, Lewis G. Carpenter, Paolo L. Mennea, Christopher Holmes, James C. Gates, \\ Peter G. R. Smith \\ Optoelectronics Research Centre, Southampton University, Southampton, SO17 1BJ, UK
}

\begin{abstract}
An optomechanical dual cantilever device has been fabricated with applications as a displacement sensor and variable attenuator. A novel fabrication approach using a precision dicing saw has benefits for fabrication time, cost and energy consumption. The displacement sensor sensitivity is $0.8 \mathrm{~dB} /$ micron and a suppression ratio of $25 \mathrm{~dB}$ is obtained when the device is used as an attenuator.
\end{abstract}

Keywords: Dual cantilever, Force sensing, MOEM's, Optical waveguide

\section{INTRODUCTION}

Various types of opto-mechanical sensing devices have been reported in the literature. The cantilever is a common geometry for characterizing surfaces in AFM and other surface profilometers. Mechanical motion of the cantilever can be converted to an optical signal by different schemes. One category of sensor works by a mirror mounted on the cantilever deflecting a beam of light. ${ }^{1}$ If the components for optical sensing are previously integrated into the cantilever structure, this can be beneficial for both the compactness and performance of the device. Holmes et al demonstrated the combination of an optical waveguide with a single glass cantilever for force sensing. ${ }^{2}$ Currently, photolithographic techniques are highly popular in the fields of integrated optics and MOEM's. These techniques have many benefits, such as parallel processing and small feature size, however they are not capable of generating all the structures that maybe required in an optomechanical device. Physical dicing has already been shown to produce low roughness optical facets. ${ }^{3}$ This paper presents a physical dicing technique is used for device fabrication which gives rapid material removal and good surfaced roughness.

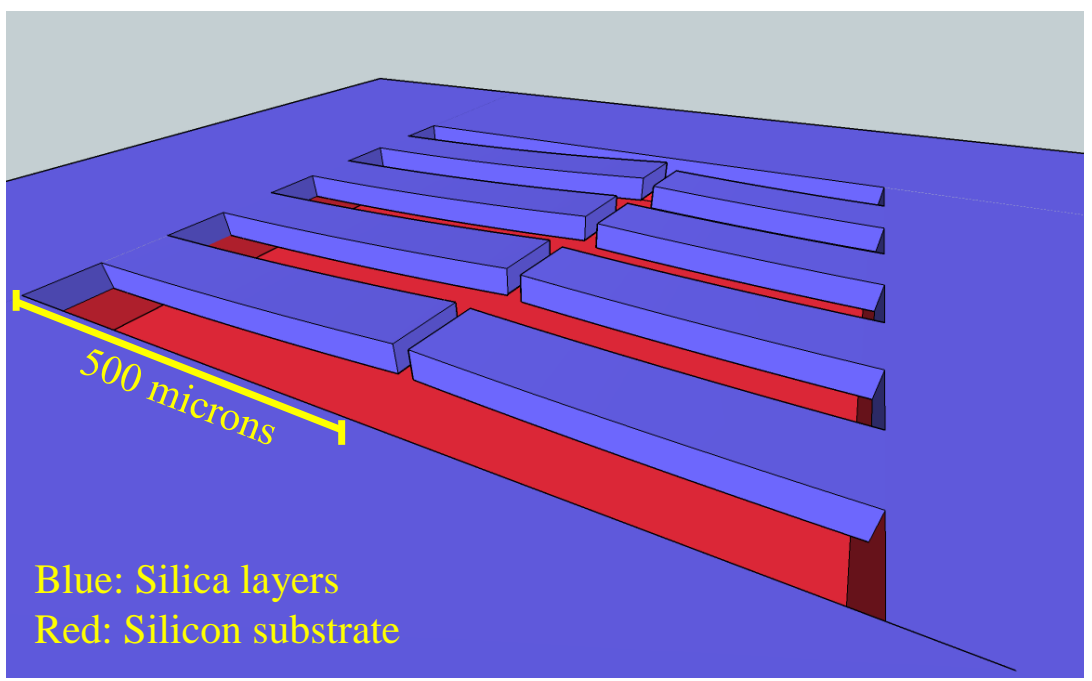

Figure 1 Graphic showing dual cantilevers following etch release 


\section{FABRICATION}

The graphic in Figure 1 shows the basic scheme for the device. The device was fabricated using a combination of physical machining techniques and wet etching. Flame Hydrolysis Deposited (FHD) glass on a silicon chip is used as the substrate into which the waveguides are written ${ }^{4}$, and the cantilevers defined. Planar silica waveguides are an established technology in the sensing and telecommunication industries. A thermally grown oxide on the silicon acts as the underclad to the waveguide. The core layer consists of FHD glass doped with germanium to provide photosensitivity, essential for the writing process. A cladding layer is then deposited which is doped with phosphorous and boron to give it a lower consolidation temperature to the core. Due to the glass layers all having different thermal expansion coefficients than the silicon, they are all under residual compressive stress. The stress will vary throughout the glass due to the different doping levels and also consolidation temperatures. As will be discussed later, this results in bending of the cantilevers following the etch release. Seven grooves through the glass into the silicon were defined using a commercial Loadpoint Microace 3000 Dicing saw. Each groove is approximately $1 \mathrm{~mm}$ long, and 100 microns wide. A nickel bonded blade impregnated with 4000 grit diamond abrasive was used. The saw was used in plunge cut mode, which consists of the blade coming vertically downwards into the substrate. As the plunge cut mode is susceptible to blade breakage and substrate chipping, process optimization was required to avoid this. Due to the circular blade, the resultant grooves are crescent shaped with the silicon only being exposed at the center where the grooves is deepest as illustrated in Figure 2. This will be important later as it allows for selective undercutting of the silicon substrate.

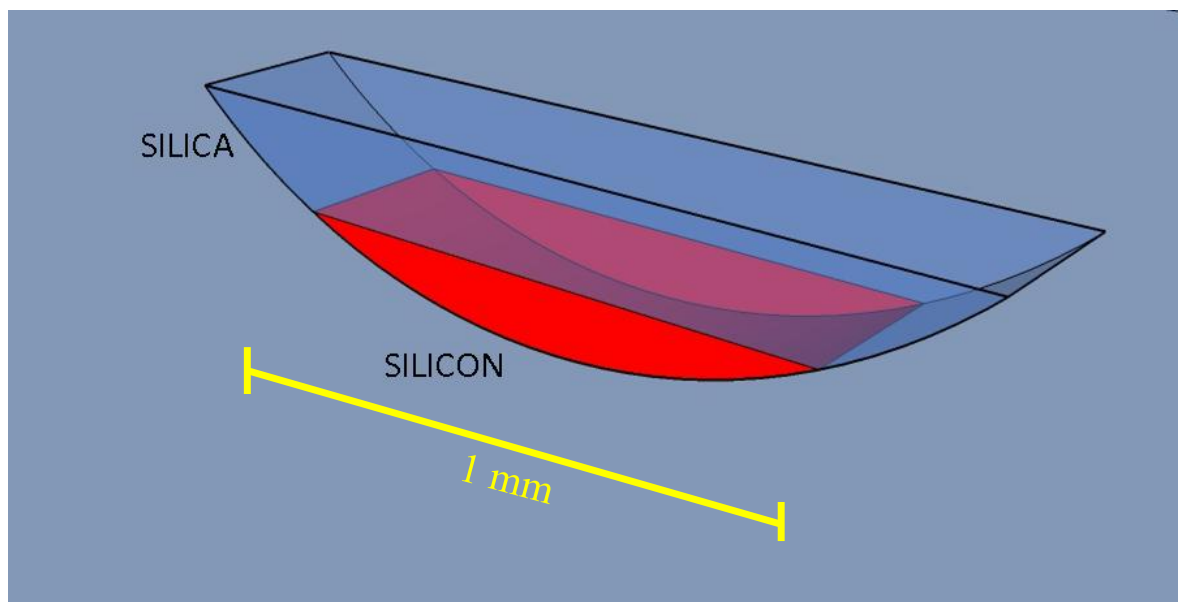

Figure 2 Graphic showing the groove following a plunge cut. Red indicates the exposed silicon. The grooves are 100 micrometres wide and $\sim 70$ micrometres deep at their centres.

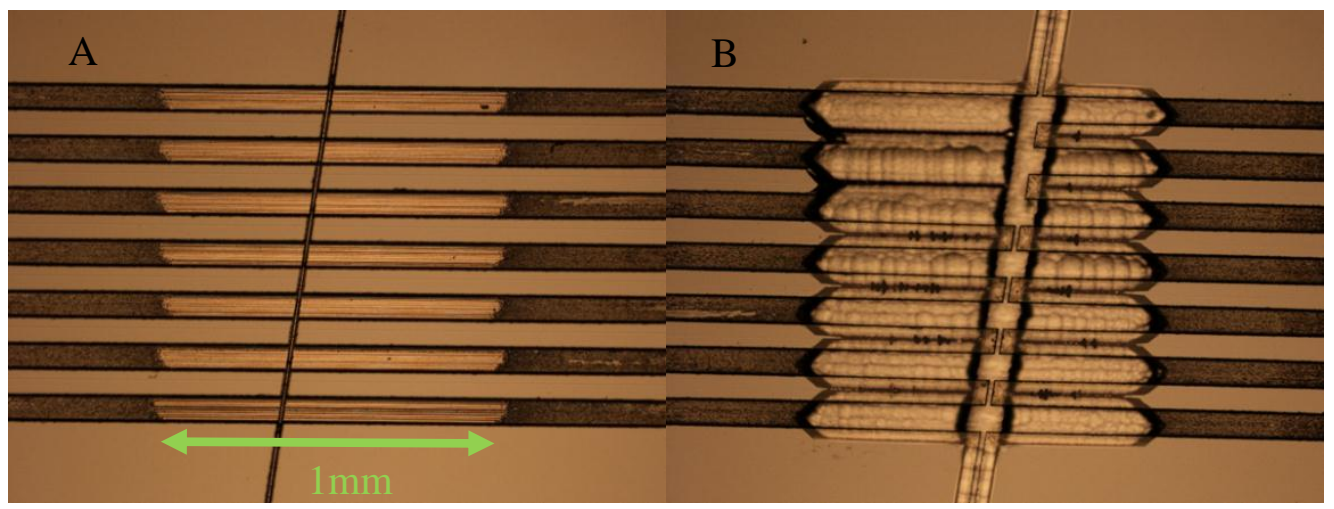

Figure 3 shows optical microscope images of the cantilevers before etching (A) and after etching (B). Note that rough appearance and broadening of the grooves in the areas where the silicon was exposed. 
An additional groove is diced with a 10 micron width blade at an angle of 8 degrees from perpendicular to the previous grooves. The angled end facets ensure that any reflected light is not guided, which would otherwise cause etalon fringes in the measured spectrum. A microscope image of the machined device is shown in Figure 3(a).

The direct UV writing process is used to define the waveguides and Bragg gratings. Using the fluorescence from the sample, the waveguides and Bragg gratings can be precisely aligned to the milled grooves. It is important that the waveguides are sufficiently central in the cantilevers to avoid the mode interacting with the cantilever sidewalls which would cause additional loss.

The cantilevers are undercut using a potassium hydroxide wet etch which selectively removes the silicon. A $25 \% \mathrm{KOH}$ solution at $75^{\circ} \mathrm{C}$ was used etched for approximately 5 hours. A microscope image and scanning electron microscope image of the finished device are shown in Figure 3(b) and Figure 4 respectively.

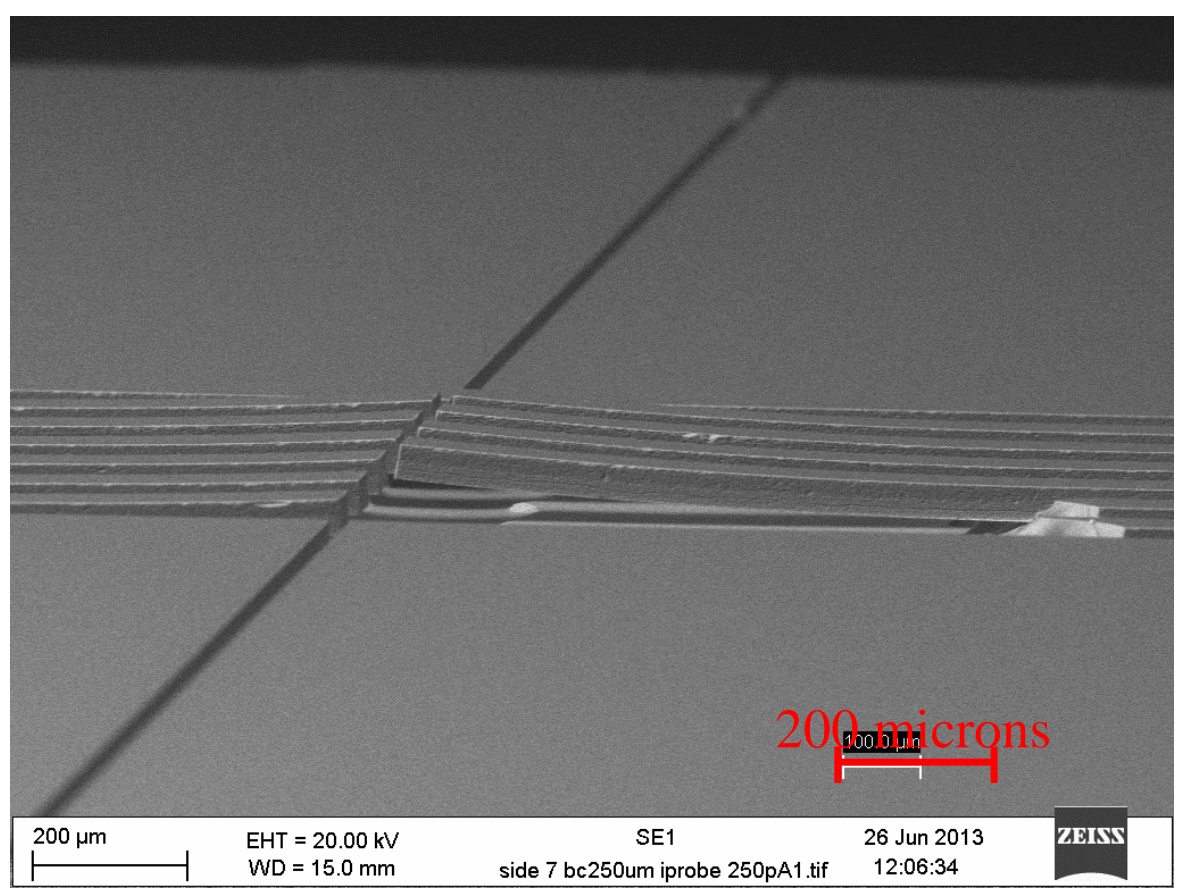

Figure 4 SEM image of the etched cantilever device. Note that the cantilevers rise out of the plane of the device due to the stress gradients in the doped glass layers.

A Zescope white light interferometer was used to measure the deflection of the cantilevers following the etch release and the data is shown in Figure 5. This confirmed that the form of the deflection was independent of the cantilever length as expected. It can be seen that for the most evenly matched cantilevers in set 4 there is still a misalignment of $5 \mu \mathrm{m}$ between the cores. Two of the cantilever sets were damaged during the etching process and as such no data is presented for these, these are visible in Figure 4 on the right hand side. 


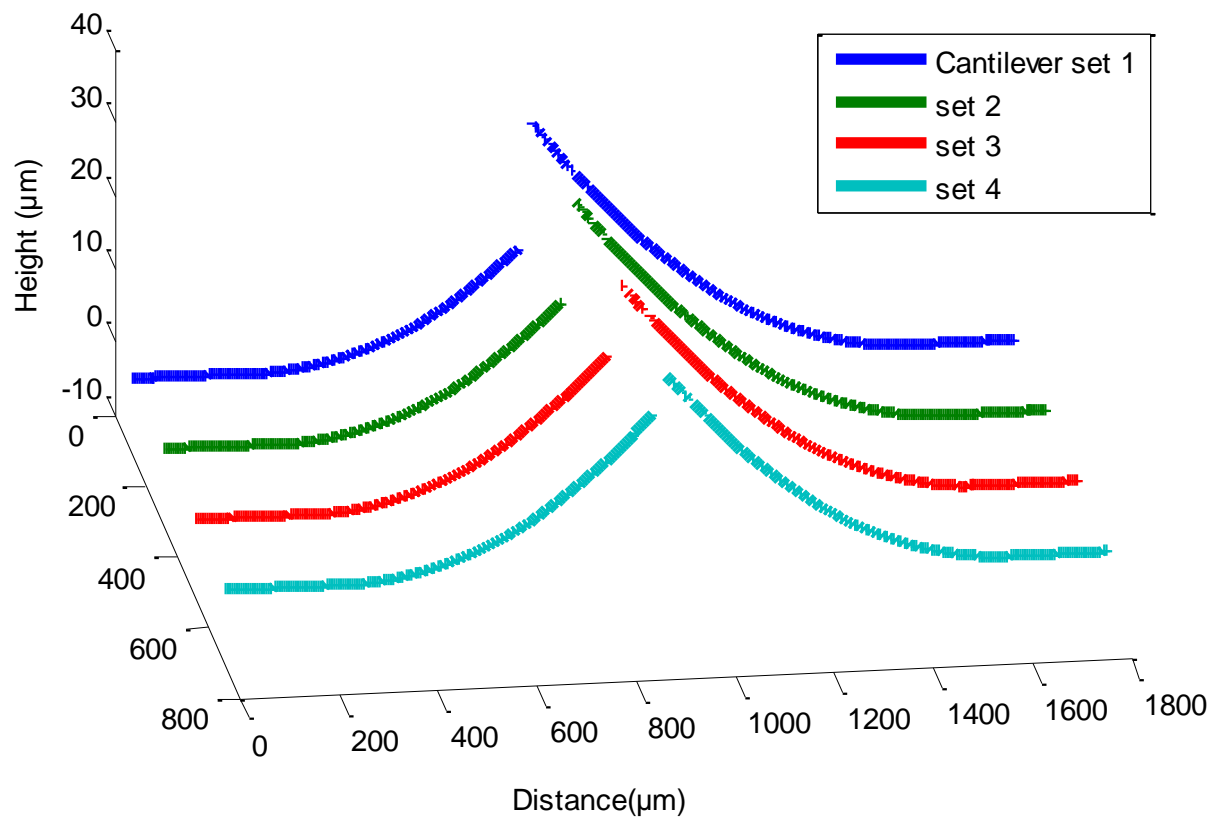

Figure 5 White light interferometer data showing out-of-plane deflection of the cantilevers measured using the Zescope.

\section{CHARACTERIZATION}

The optical spectrum of the device was measured in reflection using a broadband ASE source and Optical Spectrum Analyzer (OSA). The two orthogonal polarization modes can be measured separately. Figure 6 shows the reflection spectrum of the two polarization states. 


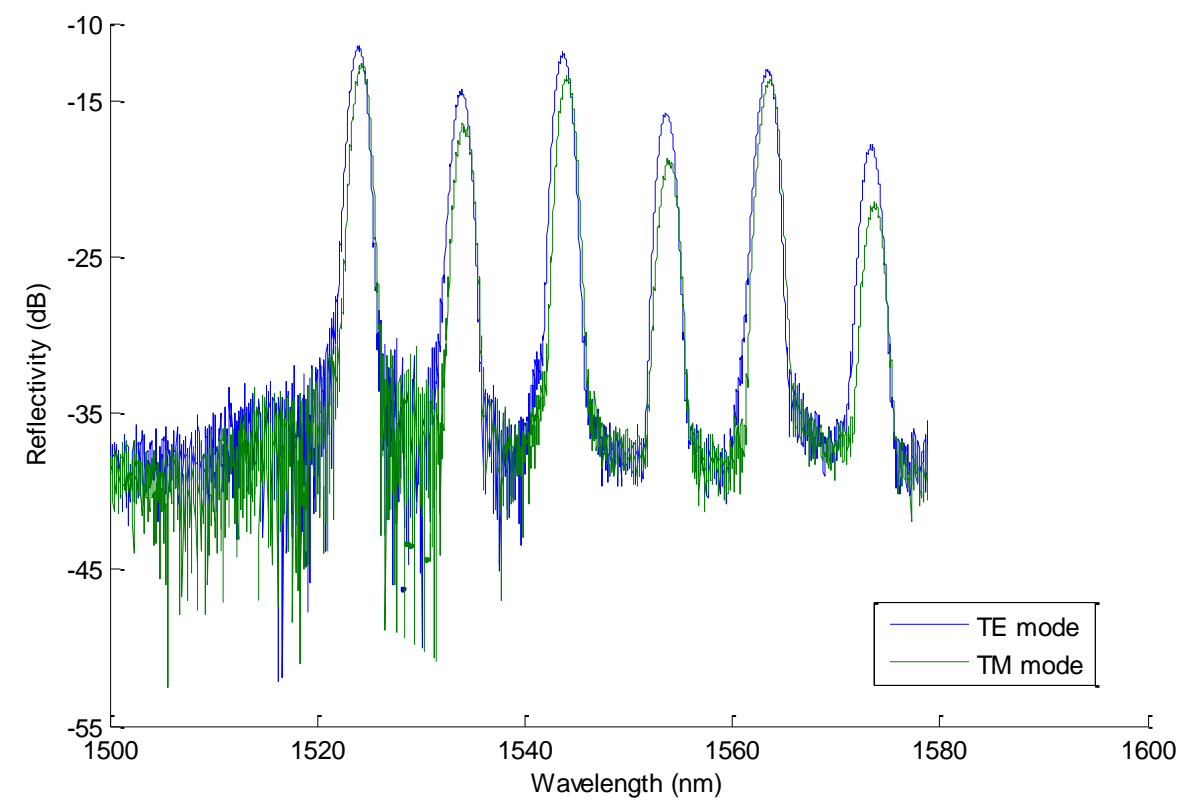

Figure 6 Spectra before etching showing loss due to Fresnel reflection at groove. The gratings at 1520, 1540 and $1560 \mathrm{~nm}$ are on the coupled side of the central groove, and the other gratings are on the far side. This shows the spectral response of the device to a broadband source and can resolve the two orthogonal polarizations

To simulate the operation of the device as a displacement sensor a cleaved optical fiber mounted on a translation stage was used to actuate the cantilevers. The small diameter of the optical fiber ensures that the deflection is applied close to the tips of the cantilevers. Two modes of actuation are possible: to push both cantilevers simultaneously or to only deflect one, diagrammatically shown in Figure 7.
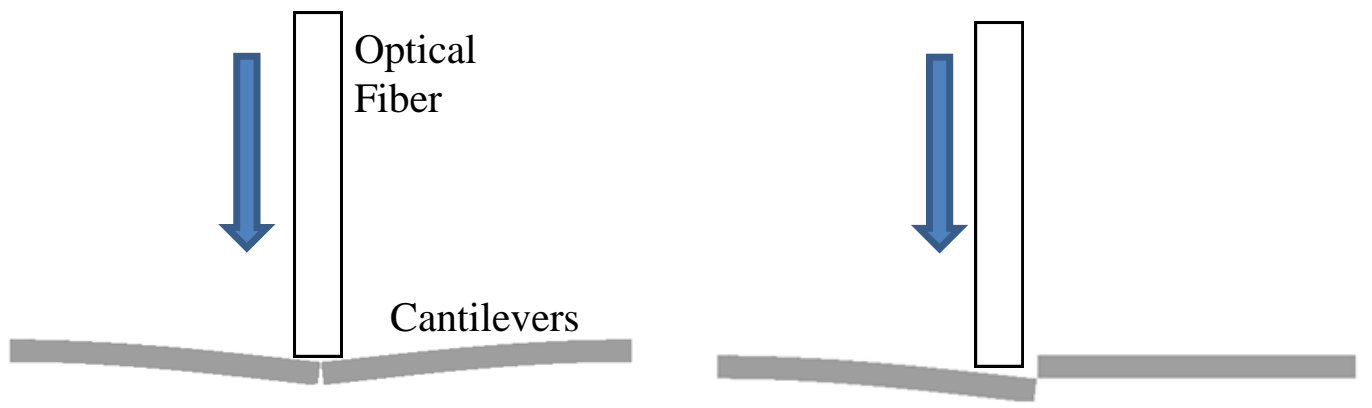

Figure 7 showing the two types of actuation that were possible. Pushing a single cantilever will give a maximum when the end facets of both cantilevers are at the same height, although in position there will be loss due to the relative angle between the waveguides

The device was mounted onto a Thorlabs translation stage which allowed $\mathrm{X}, \mathrm{Y}$ and $\mathrm{Z}$ positioning to better than micron level accuracy. Figure 8 shows a comparison of the spectra for the actuated and rest states. When the device is actuated the Bragg peaks from the side of the device opposite to the fiber-optic pigtail side become visible. There is an increase of approximately $10 \mathrm{~dB}$ in the background level due to increased Fresnel reflection from the second cantilever facet. 


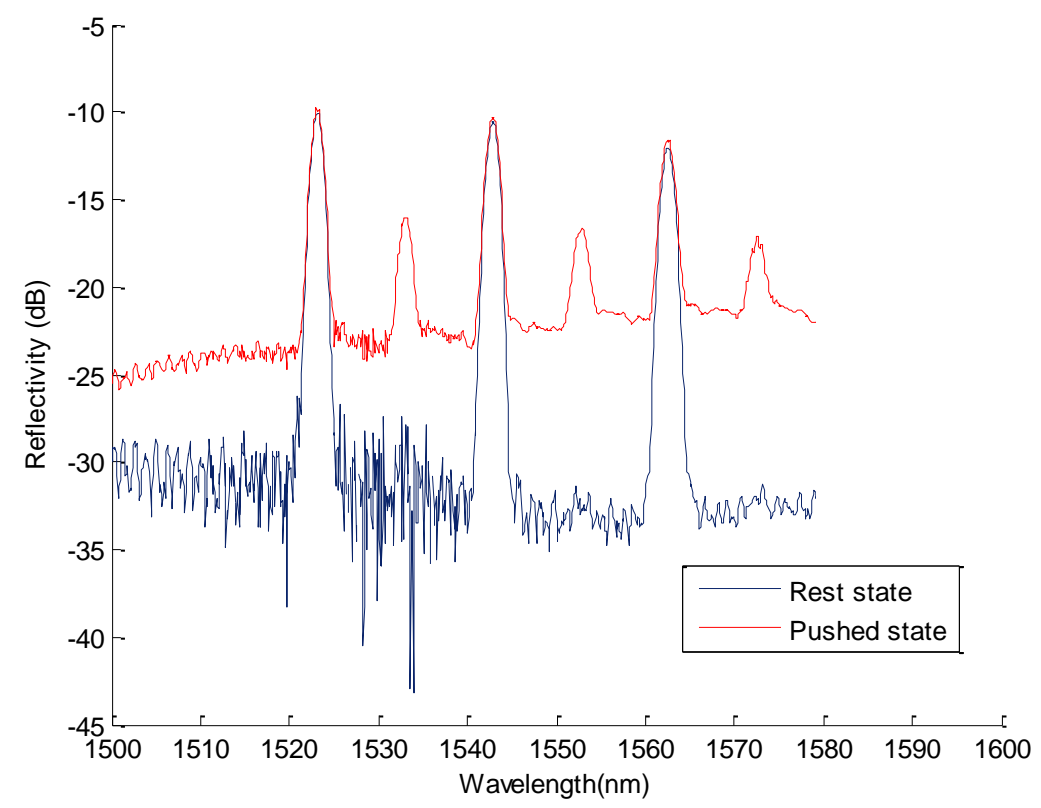

Figure 8 shows the measured spectra of the device in the rest state, and a pushed state. Actuating the devices increases the reflectivity of Bragg gratings opposite to the coupling side. As can be seen it also increases the background level by approximately $10 \mathrm{~dB}$.

The optical coupling between the cantilevers is determined by three factors:- the angle between the facets, the transverse translation, and the longitudinal translation. By measuring the transmitted intensity it is possible to measure the coupling. However by using the Bragg grating reflectivities it is possible to measure them in a way which is insensitive to fluctuations in source power and requires only coupling to one waveguide end ${ }^{5}$. Peak intensities are fitted to Gaussian apodized Bragg gratings on opposite sides of the central cavity to give a ratio dependent on the optical coupling. 


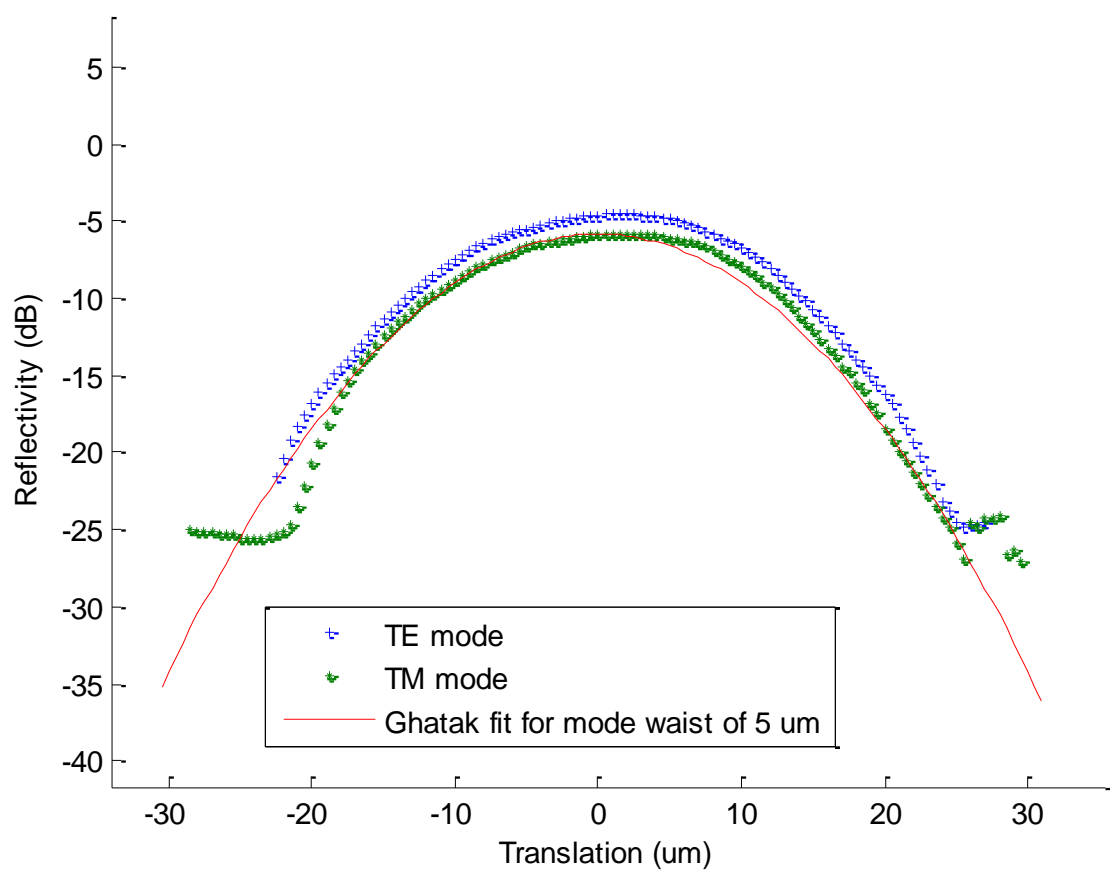

Figure 9 The actuation of both cantilevers has a geometric similarity to the problem of misalignment in fibre-optic coupling. For these devices the angular misalignment dominates over longitudinal and translational misalignment.

Figure 9 shows that the optical coupling goes through a maximum when the angle between the two cantilevers is at a minimum. There is a suppression ratio of $\sim 20 \mathrm{~dB}$ for both the TE mode and TM mode. This corresponds to an average sensitivity of $0.8 \mathrm{~dB} /$ micrometer over the central range.

The device was also characterized in transmission by coupling a fiber optic to both waveguide ends. A tunable laser was used as the source and was tuned to a wavelength between the Bragg peaks. The experiment was performed both in air and immersed in a refractive index oil of 1.5 . A suppression ratio of $\sim 25 \mathrm{~dB}$ was measured when the refractive index oil was used. The transmission loss is shown in Figure 10. 


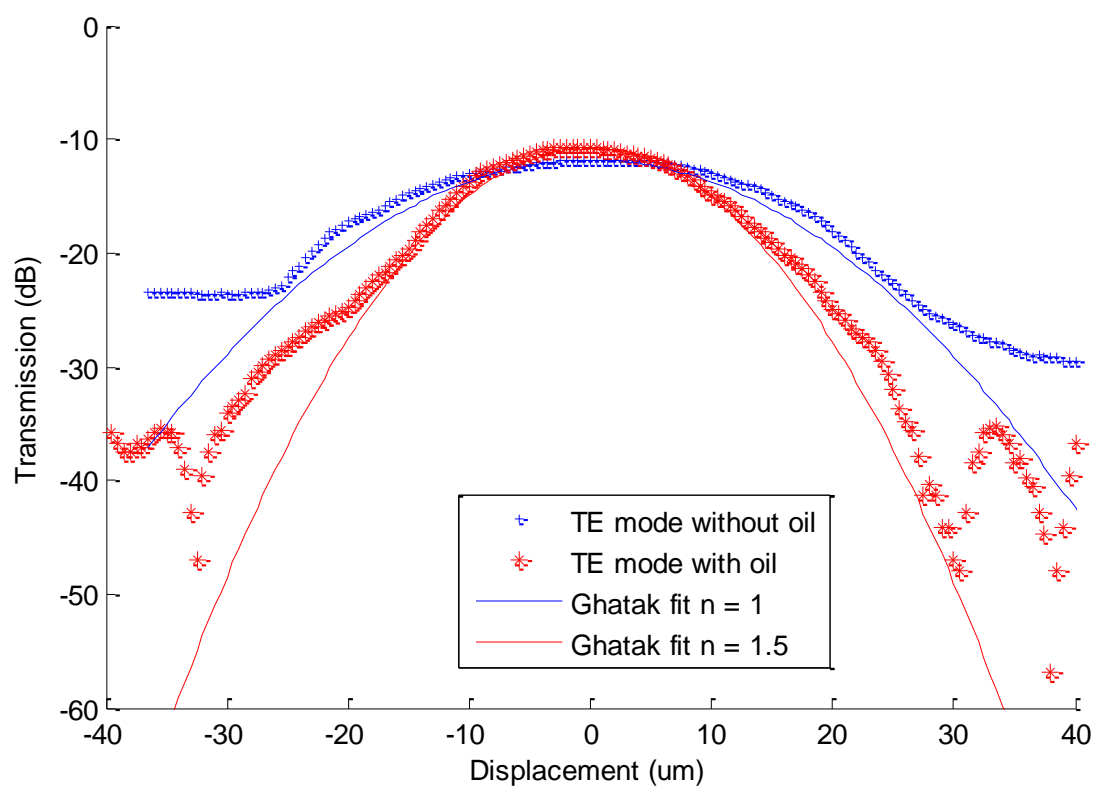

Figure 10 Transmission from actuation of both cantilevers showing transmission both with and without refractive index oil.

The geometry is closely related to the problem of loss due to angular misalignment between two fibers. Here the misalignment angle is taken to be twice the angle of rotation of a single cantilever. ${ }^{6}$

$$
\alpha_{a}(d B)=4.34\left(\frac{\pi n_{l} w \theta}{\lambda_{0}}\right)
$$

This is derived from the overlap integral of the mode exiting one fiber to the mode of the second fiber.

The mode size $w$ is approximated as 5 microns which is similar to SMF28 single mode fiber. As can be seen from Figure 9 the approximation fits well to the measured data, and shows potential for these devices to be used in switching configurations.

\section{CONCLUSION}

In this work a new type of dual-cantilever microstructure has been demonstrated which can act as either a displacement sensor or a variable attenuator. The fabrication procedure is unconventional for MOEM structures and allows rapid prototyping without lithographic facilities. The mechanical-optical behavior of the device is well described by fiber optic angular misalignment coupling theory. The use of Bragg gratings allows quantitative measurement of the loss and suppression ratio of the device which was found to be $\sim 20 \mathrm{~dB}$ for both the TE mode and for the TM mode. When measured with a transmission setup a suppression ratio of $\sim 25 \mathrm{~dB}$ was measured for the TE mode with the device immersed in refractive index oil.

\section{REFERENCES}

1. Meyer, G. \& Amer, N. M. Novel optical approach to atomic force microscopy. Appl. Phys. Lett. 53, 1045 (1988). 
2. Holmes, C., Carpenter, L. G., Rogers, H. L., Gates, J. C. \& Smith, P. G. R. Quantifying the optical sensitivity of planar Bragg gratings in glass micro-cantilevers to physical deflection. J. Micromechanics Microengineering 21, 035014 (2011).

3. Carpenter, L. G. et al. Low optical-loss facet preparation for silica-on-silicon photonics using the ductile dicing regime. J. Phys. D. Appl. Phys. 46, 475103 (2013).

4. Emmerson, G. D. et al. Fabrication of directly UV-written channel waveguides with simultaneously defined integral Bragg gratings. Electron. Lett. 38, 1531-1532 (2002).

5. Rogers, Helen L. Ambran, Sumiaty Holmes, Christopher Smith, Peter G. R. Gates, J. C. In situ loss measurement of direct UV-written waveguides using integrated Bragg gratings. Opt. Lett. 35, 2849-2851 (2010).

6. Ghatak, A. \& Thyagarajan, K. An Introduction to Fiber Optics. 154 (Cambridge University Press, 1998). 